\title{
Synchronisation and Equalisation of an FBMC/OQAM System by a Polynomial Matrix Pseudo-Inverse
}

\author{
Amr A.A. Nagy and Stephan Weiss \\ Department of Electronic and Electrical Engineering \\ University of Strathclyde, Glasgow G1 1XW, Scotland \\ Email:\{amr.a.nagy,stephan.weiss\}@ strath.ac.uk
}

\begin{abstract}
Filter bank-based multicarrier using offset quadrature amplitude modulation (FMBC/OQAM) systems suffer from inter-symbol-interference (ISI) and cochannel interference (CCI) if the transmission channel is dispersive, or simply if the overall system is not synchronised. This paper considers the solution to an equaliser that can mitigate both ISI and CCI by describing the equivalent transmultiplexed channel including the filter banks as a polyphase matrix of transfer functions, and by using polynomial matrix algebra to equalise this system. We demonstrate that the reduced-rank nature of this channel matrix requires a polynomial matrix pseudoinverse, and demonstrate how the overall FBMC/OQAM system is orthogonalised, thus removing ISI and ICI.
\end{abstract}

Keywords-FMBC/OQAM; equalisation; polynomial matrix factorisation.

\section{INTRODUCTION}

Multicarrier modulation techniques have been central to the development of wideband wireless communications standards for more than a decade. Within fifth generation communications systems, particularly filter bank-based multicarrier (FMBC) techniques are emerging a candidates radio front-ends [1]-[3]. Such FBMC systems are popular because offer increased robustness against synchronisation errors compared to e.g. orthogonal frequency division multiplexing (OFDM) systems [4]. The approach in [1] of combining FBMC with offset quadrature amplitude modulation (OQAM), refered to as an FBMC/OQAM system, has attracted attentention as it is a critically sampled transceiver without any guard intervals, and hence theoretically is able to maximise spectral efficiency [2].

FBMC/OQAM consists of two main blocks: a synthesis filter bank (SFB) in the transmitter and an analysis filter bank (AFB) in the receiver. The prototype filters are much more frequency-selective than e.g. in OFDM, which can significantly reduce the out-ofband emission and allow a flexible spectrum usage [5]. Since a critically sampled DFT-modulated filter bank itself cannot be perfectly reconstructing, incorporating OQAM can lead to perfect reconstruction even under critical sampling and the lack of guard bands. The latter enable maximum spectrum efficiency [6], but as a consequence FBMC/OQAM must permit an overlap of spectra between at least adjacent subcarriers.

Because of this spectral overlap, in a dispersive environment or an unsynchronised FBMC/OQAM transmission, each subcarrier in the receiver is affected by interference from at least adjacent subcarriers, resulting not just in inter-symbol (ISI) but also inter-channel interference $(\mathrm{CCI})$. The mitigation of this interference has received considerable attention, see e.g. [7]-[14].

This paper investigated the joint equalisation of a FBMC/OQAM system based on the same concept of [15], where leakage between adjacent channels necessitates the use of cross-terms rather than a pure perband processing. Similar to [13], [14], we use a linear algebraic approach to the equalisation of this broadband system, but here rely on the system description by polynomial matrices, which leads us to the novel definition of a polynomial matrix pseudo-inverse to address the FBMC/OQAM interference mitigation.

Our paper is organised as follows: in Sec. II, a general structure of the FBMC/OQAM system is introduced. The channel polynomial matrix and its inverse are derived in Sec. III and IV, with a rank deficient requiring the consideration of a polynomial pseudoinverse in Sec. V.

\section{FMBC/OQAM STRUCTURE}

FMBC/OQAM consists of two main components: an inner filter bank, and an outer OQAM system, as shown in Fig. 1. In the inner system, a filter bank transmultiplexes a vector $\mathbf{u}[n] \in \mathbb{C}^{M}$ over a channel with impulse response $c[\nu]$ to generate a demultiplexed output $\mathbf{x}[n]$, with the time index $n$ running $\frac{M}{2}$ times slower than the index $\nu$. The outer system consists of OQAM staggering and de-staggering blocks, which map a transmit vector $\mathbf{d}[\ell] \in \mathbb{C}^{M}$ onto $\mathbf{u}[n]$, with index $\ell$ now running $M$ times slower than $\nu$, and at half the speed of index $n$. The de-staggering then generates an output $\hat{\mathbf{d}}[\ell] \in \mathbb{C}^{M}$ from $\mathbf{x}[n]$. 


\section{A. Offset $Q A M$}

The functions of the OQAM system are carried out by two blocks: OQAM pre-processing in the transmitter and OQAM post-processing in the receiver. The input to the OQAM pre-processing are $M$ parallel QAM signals $\mathbf{d}[\ell]$, where $M$ is the number of subchannels. The complex-valued QAM symbols in $\mathbf{d}[\ell]$ are demultiplexed into their real and imaginary components alternatingly, as shown in Fig. 2. The sequence of the components is dependent on the subchannel index, and alternates between adjacent channels.

The reverse process is executed by the OQAM postprocessing, where the signals in $\mathbf{y}[n]$ are demultiplexed and imaginary and real components are alternatingly discarded. The discarding operation again depends on the subchannel index, as shown in Fig. 2.

\section{B. Filter Bank Multicarrier System}

The inner component of the FBMC/OQAM transceiver is an $M$-channel DFT filter bank operated as a transmultiplexer, with the analysis filter bank (ASB) on the receiver side and the synthesis filter bank (SFB) in the transmitter as shown in Fig. 1. Since the input $\mathbf{u}[n] \in \mathbb{C}^{M}$ and output $\mathbf{x}[n] \in \mathbb{C}^{M}$ are operated at an $\frac{M}{2}$-fold slower time index than the multiplexed data transmitted over the channel $c[\nu]$, we choose a polyphase representation below.

In the receiver, the analysis filter bank (ASB) is formed by a DFT filter bank. In polyphase notation, the input is first demultiplexed by a serial to parallel converter ( $\mathrm{s} / \mathrm{p}$ ) before feeding into a polyphase analysis matrix $\boldsymbol{H}(z): \mathbb{C} \rightarrow \mathbb{C}^{M \times M / 2}$, as seen in Fig. 3. This matrix consists of a multiple-input multiple-output system, which can be factored into a network of polyphase components of the filter bank's prototype filter, followed by the modulating transform, i.e. a DFT matrix.

In the transmitter, a matching DFT synthesis filter bank can also be expressed in polyphase notation. Here, the upsampling by $\frac{M}{2}$ is swapped with the filtering operation, such that a polyphase synthesis matrix first operates on the $M$ inputs of $\mathbf{u}[n]$, before $\frac{M}{2}$ outputs are multiplexed by a parallel to serial converter, to generate the signal to be transmitted over the channel $c[\nu]$. The polyphase synthesis matrix is $\boldsymbol{H}^{\mathrm{P}}(z): \mathbb{C} \rightarrow \mathbb{C}^{M / 2 \times M}$, which relates to the polyphase analysis matrix by a parahermitian operation, whereby $\boldsymbol{H}^{\mathrm{P}}(z)=\boldsymbol{H}^{\mathrm{H}}\left(1 / z^{*}\right)$,

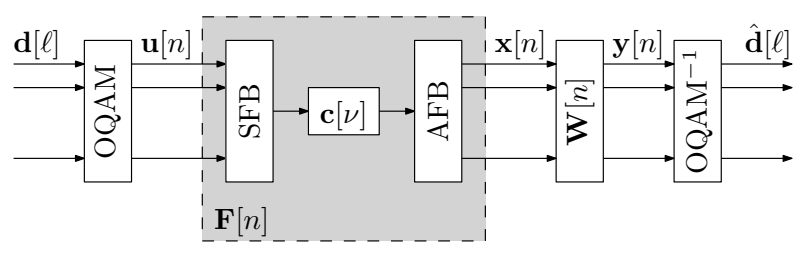

Fig. 1. FBMC-OQAM system model with equaliser $\mathbf{W}[n]$.

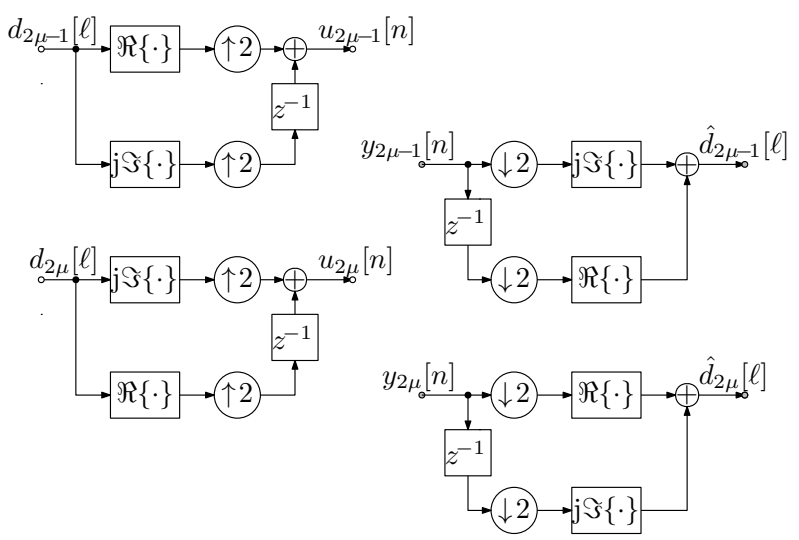

Fig. 2. OQAM (left) preprocessing and (right) postprocessing [16] for two adjacent subchannels, with $\mu=1 \ldots \frac{M}{2}$.

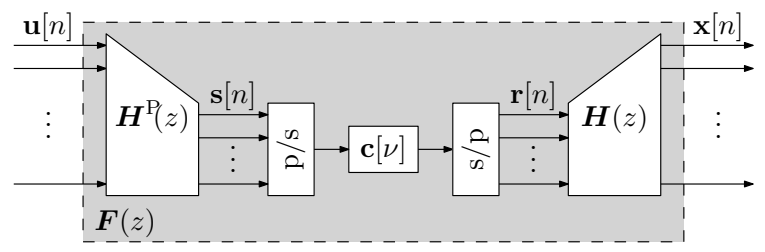

Fig. 3. Inner components of the FBMC/OQAM system with synthesis and analysis filter banks and the channel impulse response $c[\nu]$.

i.e. consisting of a Hermitian transposition and time reversal.

The design of the prototype filter plays a crucial role in generating the sub-channels filters in both SFB and AFB [16]. The prototype filter used in this paper is based on the design presented in [17], which due to its high frequency selectivity, ensures that the crosstalk between non-adjacent subchannels is kept sufficiently small. Aliasing between adjacent subchannels cannot be entirely suppressed by the DFT filter bank, but is removed due to the OQAM arrangement. However, any delay or time-dispersion of the channel $c[\nu]$ will destroy orthogonality and result in inter-symbol (ISI) and co-channel-interference (CCI) in the output $\hat{\mathbf{d}}[\ell]$. We therefore explore a synchronisation and equalisation approach next.

\section{EQualisation OF FBMC System}

\section{A. Equivalent Transmission System}

We first aim to describe the subchannel transfer functions of the inner FBMC system in in Figs. 1 and 3 which can be represented as a polynomial matrix $\boldsymbol{F}(z): \mathbb{C} \rightarrow \mathbb{C}^{M \times M} \bullet-\circ \mathbf{F}[n]$. Considering the channel impulse response $c[\ell]$ together with the multiplexer and demultiplexer in Fig. 3, this subsystem can be characterised by a pseudo-circulant polyphase matrix 


$$
\begin{aligned}
C(z) & : \mathbb{C} \rightarrow \mathbb{C}^{M / 2 \times M / 2}[18], \\
\mathbf{C}(z) & =\left[\begin{array}{cccc}
C_{0}(z) & C_{1}(z) & \ldots & C_{\frac{M}{2}-1}(z) \\
z^{-1} C_{\frac{M}{2}-1}(z) & C_{0}(z) & \cdots & C_{\frac{M}{2}-2}(z) \\
\vdots & & \ddots & \vdots \\
z^{-1} C_{1}(z) & \cdots & z^{-1} C_{\frac{M}{2}-1}(z) & C_{0}(z)
\end{array}\right],
\end{aligned}
$$

which comprises the $\frac{M}{2}$ polyphase components $C_{\mu}(z)$ of the channel transfer function $C(z) \bullet \circ c[\ell]$. These are defined as

$$
C(z)=\sum_{\mu=0}^{\frac{M}{2}-1} C_{\mu}\left(z^{\frac{M}{2}}\right) z^{-\mu}
$$

or alternatively can be obtained from the channel impulse $c[\ell]$ via $C_{\mu}(z)=\sum_{\ell} c\left[\frac{M}{2} \ell+\mu\right] z^{-\ell}$.

Using the polyphase synthesis and analysis matrices for the FBMC system shown in Fig. 3, the equivalent system $\boldsymbol{F}(z)$ can be obtained as

$$
\boldsymbol{F}(z)=\boldsymbol{H}(z) \boldsymbol{C}(z) \boldsymbol{H}^{\mathrm{P}}(z) .
$$

If $F_{i j}(z)$ is the element in the $i$ th row and $j$ th column of $\boldsymbol{F}(z)$ in (2), it represents the transfer function between the $j$ th signal in the input vector $\mathbf{u}[n]$ and the $i$ th signal in the output vector $\mathbf{y}[n]$. Thus, the diagonal elements $F_{m m}(z), m=1 \ldots M$, represent the per-subchannel transfer functions, while due to the frequency selectivity, cross-talk is restricted to adjacent bands only such that

$$
\begin{gathered}
F_{i j}(z) \approx 0, \quad \forall\left|\bmod _{M} i-\bmod _{M} j\right|>1, \\
i, j=1 \ldots M .
\end{gathered}
$$

The structure of $\boldsymbol{F}(z)$ is therefore tri-diagonal, including non-zero corner elements irrespective of the channel polyphase matrix $C(z)$.

Example. An example of $\boldsymbol{F}(z)$ for a distorted FBMC/OQAM system with $M=4$ subchannels is obtained for an arbitrary channel $c[\ell]$ of length 4 . The components of $\boldsymbol{F}(z)$ are determined by channel sounding, and are depicted in Fig. 4.

\section{B. Equalisation of FBMC System}

Because the first stage of the OQAM postprocessing in Fig. 2 alternatingly discards the real and imaginary parts of $y_{m}[n], m=1 \ldots M$, achieving a transparent system such that $\hat{\mathbf{d}}[\ell]=\mathbf{d}[\ell]$ in Fig. 1 does not necessarily require $\mathbf{y}[n]=\mathbf{u}[n]$. However, a sufficient criterion for the overall synchronisation and equalisation of the FBMC/OQAM system is achieved if

$$
\mathbf{y}[n] \approx \mathbf{u}[n-2 \Delta \tau],
$$

where $\Delta \tau \in \mathbb{N}$ represents an arbitrary delay. Note that ultimate delay between $\mathbf{y}[n]$ and $\mathbf{u}[n]$ must be even to guarantee synchronisation with $\hat{\mathbf{d}}[\ell] \approx \mathbf{d}[\ell-\Delta \tau]$. The condition (4) does not exploit the alternate discarding of real and imaginary parts of $\mathbf{y}[n]$ in the OQAM postprocessing, but leads to a simple solution below.

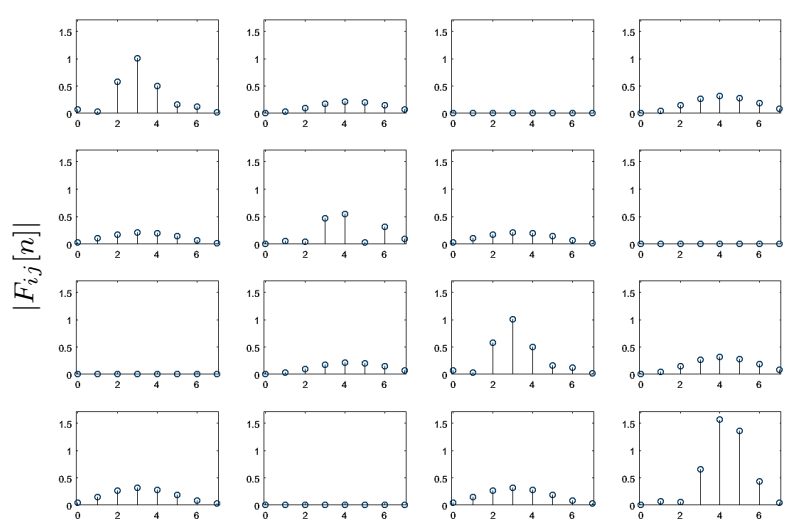

time index $n$

Fig. 4. Example of a $4 \times 4$ polynomial channel matrix $\boldsymbol{F}(z)$.

Since an equaliser must cancel ICI, it requires crosstalk terms akin to $\boldsymbol{F}(z)$. Therefore, in this paper we propose a polynomial matrix equaliser $\boldsymbol{W}(z)$ inserted between the AFB and OQAM post-processing in the receiver, as shown in Fig. 1. This assumes that $\boldsymbol{F}(z)$ is available - it can either be obtained via channel sounding directly, or synthesised according to (2) based on the measured channel impulse response $c[\nu]$ with $\boldsymbol{H}(z)$ known. By calculating a polynomial singular value decomposition (PSVD, [19]-[21]) $\boldsymbol{F}(z)=$ $\boldsymbol{U}(z) \boldsymbol{\Sigma}(z) \boldsymbol{V}^{\mathrm{P}}(z)$, we can determine $\boldsymbol{W}(z)$ as

$$
\boldsymbol{W}(z)=\boldsymbol{F}^{-1}(z)=\boldsymbol{V}(z) \boldsymbol{\Sigma}^{-1}(z) \boldsymbol{U}^{\mathrm{P}}(z),
$$

where $\boldsymbol{U}(z)$ and $\boldsymbol{V}(z)$ are paraunitary matrices such that e.g. $\boldsymbol{U}(z) \boldsymbol{U}^{\mathrm{P}}(z)=\boldsymbol{U}^{\mathrm{P}}(z) \boldsymbol{U}(z)=\mathbf{I}$. The matrix $\boldsymbol{\Sigma}(z)$ is diagonal,

$$
\boldsymbol{\Sigma}(z)=\operatorname{diag}\left\{\sigma_{1}(z) \ldots \sigma_{M}(z)\right\},
$$

and contains the polynomial singular values $\sigma_{m}(z)$, $m=1 \ldots M$. Therefore, the inversion of $\boldsymbol{F}(z)$ reduces to the calculation of its PSVD, and the inversion of the singular values.

\section{POlynomial MatriX InVERSION VIA PSVD}

To calculate $\boldsymbol{W}(z)=\boldsymbol{F}^{-1}(z)$, we first determine the PSVD of $\boldsymbol{F}(z)$. Three general options have been discussed in the literature: there is a direct iterative calculation [19], an iterative calculation using a polynomial QR approach [20], and the route via two polynomial eigenvalue decompositions (PEVDs) [21]. We here choose the later due to the availability of enhanced PEVD algorithms such the sequential matrix diagonalisation (SMD) approach [22].

Like other PEVD algorithms, SMD is applicable to polynomial matrices $\boldsymbol{A}(z)$ that possess the parahermitian property, such that $\boldsymbol{A}(z)=\boldsymbol{A}^{\mathrm{P}}(z)$. We form two such parahermitian matrices, and can calculate their 
their approximate PEVDs using the SMD algorithm:

$$
\begin{aligned}
& \boldsymbol{R}_{1}(z)=\boldsymbol{F}(z) \boldsymbol{F}^{\mathrm{P}}(z) \approx \boldsymbol{U}(z) \boldsymbol{S}_{1}(z) \boldsymbol{U}^{\mathrm{P}}(z), \\
& \boldsymbol{R}_{2}(z)=\boldsymbol{F}^{\mathrm{P}}(z) \boldsymbol{F}(z) \approx \boldsymbol{V}(z) \boldsymbol{S}_{2}(z) \boldsymbol{V}^{\mathrm{P}}(z) .
\end{aligned}
$$

This determines the two paraunitary matrices for (5). If $\boldsymbol{F}(z)$ is square, we expect $\boldsymbol{\Sigma}(z)=\boldsymbol{S}_{1}(z) \boldsymbol{S}^{\mathrm{P}}{ }_{1}(z)=$ $\boldsymbol{S}_{2}(z) \boldsymbol{S}^{\mathrm{P}}{ }_{2}(z)$. However, unless $\boldsymbol{\Sigma}(z)$ is constrained (e.g. to be minimum phase), it cannot be directly extracted from $\boldsymbol{S}_{1}(z)$ and $\boldsymbol{S}_{2}(z)$, and we instead need to calculate

$$
\boldsymbol{\Sigma}(z)=\boldsymbol{V}(z) \boldsymbol{F}(z) \boldsymbol{U}^{\mathrm{P}}(z)
$$

in order to determine the polynomial singular values in $\boldsymbol{\Sigma}(z)$.

To determine $\boldsymbol{\Sigma}^{-1}(z)$ requires the inversion of the polynomial singular values in (6). Since $\sigma_{m}(z)$, $m=1 \ldots M$ is generally non-minimum phase, zeros of $\sigma_{m}(z)$ inside the unit circle will lead to causal components of $\sigma_{m}^{-1}(z)$, while roots of $\sigma_{m}(z)$ outside the unit circle will result in anti-causal components of $\sigma_{m}^{-1}(z)$. The inversion can be performed by several approaches, akin to the inversion of polynomial eigenvalues in [23], such as by calculating the roots of $\sigma_{m}(z)$, determining their residues for a partial fraction expansion, and then approximating the poles by appropriately truncating their causal or anti-causal geometric series representations. Here, for an inverse $w_{m}(z)=\sigma_{m}^{-1}(z)$ of length $L$, we require $\sigma_{m}(z) w_{m}(z) \approx z^{-\Delta}$, where $\Delta$ is a suitable delay, with a rule of thumb for inverse system identification recommending $\Delta=\frac{L}{2}$ [24].

To use a least squares approximation, we define a vector $\boldsymbol{\sigma}_{m} \in \mathbb{C}^{K}$ to hold the $K$ coefficients of $\sigma_{m}(z)$, $m=1 \ldots M$. We aim to find a vector $\mathbf{w}_{m} \in \mathbb{C}^{L}$ holding the $L$ coefficients of $w_{m}(z)$ such that

$$
\mathbf{w}_{m, \text { opt }}=\arg \min _{\mathbf{w}_{m}}\left\|\left[\mathbf{I}_{L-K+1} \otimes\left(\boldsymbol{\sigma}_{m}^{\mathrm{T}} \mathbf{J}_{L}\right)\right] \mathbf{w}_{m}-\mathbf{p}\right\|_{2},
$$

with $J_{L}$ an $L \times L$ reverse identity matrix, $\otimes$ denoting a Kronecker product, and $\mathbf{p} \in \mathbb{N}^{L-K+1}$ a pinning vector containing zeros except for a one in the $\Delta$ th position. A solution can be found by a pseudo-inversion of the convolutional matrix

$$
\mathbf{A}_{m}=\mathbf{I}_{L-K+1} \otimes\left(\boldsymbol{\sigma}_{m}^{\mathrm{T}} \mathbf{J}_{L}\right),
$$

such that $\mathbf{w}_{m, \mathrm{opt}}=\mathbf{A}_{m}^{\dagger} \mathbf{p}$ [25]. Alternatively, an iterative method such as the recursive least squares (RLS) algorithm can be invoked [26].

Example. Applying a polynomial singular value decomposition as laid out above to the earlier example of an $M=4 \mathrm{FMBC} / \mathrm{OQAM}$ equivalent transmission matrix $\boldsymbol{F}(z)$ in Fig. 4 yields the matrix $\boldsymbol{\Sigma}(z)$ characterised in Fig. 5. The matrix is sufficiently diagonalised, but reveals a rank deficiency of $\boldsymbol{F}(z)$, as half of the singular values are zero. Inspecting the source model for $\boldsymbol{F}(z)$ in (2), since the inner factor $\boldsymbol{C}(z)$ is an $\frac{M}{2} \times \frac{M}{2}$ polynomial matrix, the overall rank cannot exceed $\frac{M}{2}$. To avoid

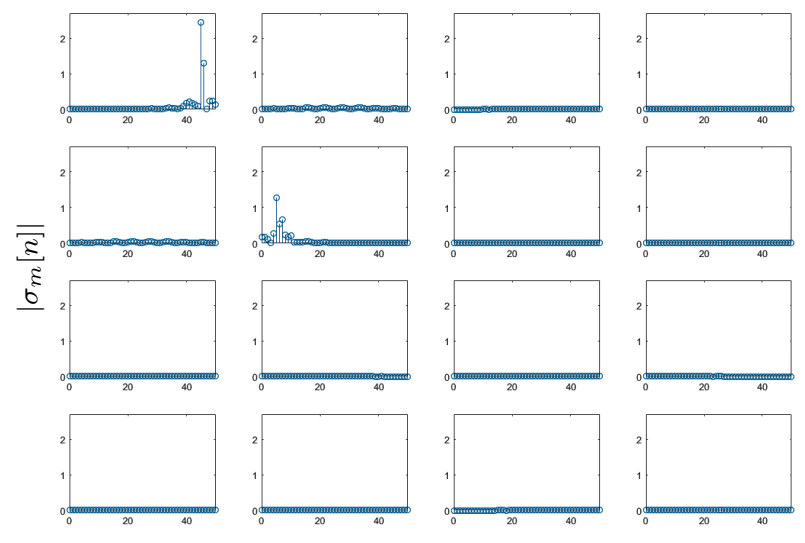

time index $n$

Fig. 5. Example of $4 \times 4$ diagonal matrix $\boldsymbol{\Sigma}(z)$, containing its diagonal terms $\sigma_{m}[n] \bullet-\circ \sigma_{m}(z)$, with all cross-terms zero or very nearly so.

the inversion of singular values that are either zero everywhere, or have zeros within a vicinity of the unit circle, we next consider a polynomial matrix pseudoinverse.

\section{Polynomial Matrix Pseudo-Inverse}

Within this paper, the aim has been to invert a square polynomial $\boldsymbol{F}(z)$. To add both robustness to the inversion approach but also to include more general, rectangular matrices $\boldsymbol{F}(z): \mathbb{C} \rightarrow \mathbb{C}^{N \times M}$, we now discuss a polynomial pseudo-inverse as an extension of the pseudo-inverse of matrices containing scalar values only [25]. For general $N \times M$ matrices, the PEVD steps (7) and (8) will yield paraunitary matrices $\boldsymbol{U}(z)$ : $\mathbb{C} \rightarrow \mathbb{C}^{N \times N}$ and $\boldsymbol{V}(z): \mathbb{C} \rightarrow \mathbb{C}^{M \times M}$. However, we will not directly invert the diagonal components of $\boldsymbol{\Sigma}(z): \mathbb{C} \rightarrow \mathbb{C}^{N \times M}$ as suggested earlier, but select

$$
\boldsymbol{\Sigma}^{-1}(z)=\operatorname{diag}\left\{w_{1}(z), \ldots w_{\min (M, N)}(z)\right\},
$$

where $w_{m}(z)$ now is the inverse of $\sigma_{m}(z)$ for finite $\sigma_{m}(z)$, and $w_{m}(z)=0$ if $\sigma_{m}(z)=0 \forall z=\mathrm{e}^{\mathrm{j} \Omega}, \Omega \in \mathbb{R}$.

For a numerical evaluation, we collate the $L$ coefficients of $w_{m}(z)$ in the vector $\mathbf{w}_{m}$, and with the earlier definitions of the singular value vectors $\sigma_{m}$, the convolutional matrix $\mathbf{A}_{m}$, and the pinning vector $\mathbf{p}$ determine

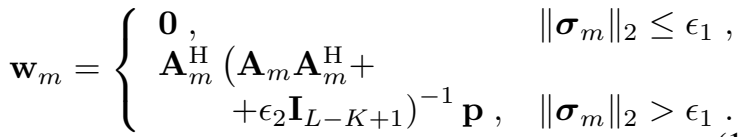

The small constant $\epsilon_{1}>0$ determines a threshold below which a singular value is treated as numerically zero. A second constant, $\epsilon_{2}$, implements a regularisation to mitigate spectral zeros in $\sigma_{m}(z)$ or zeros close to the unit circle. A larger value for $\epsilon_{2}$ may result in faster decaying responses in $w_{m}(z)$, but also introduces a bias into the original solution in (10). 


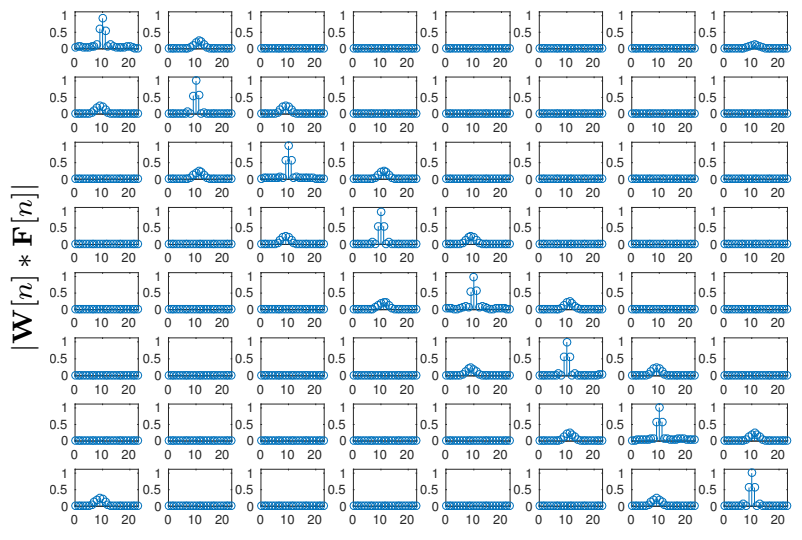

time index $n$

Fig. 6. Example for the joint channel transmission matrix $\boldsymbol{F}(z)$ and its pseudo-inverse $\boldsymbol{F}^{\dagger}(z)$ for an $M=8$ channel FMBC/OQAM system including a dispersive channel $c[\nu]$ of length 4 .

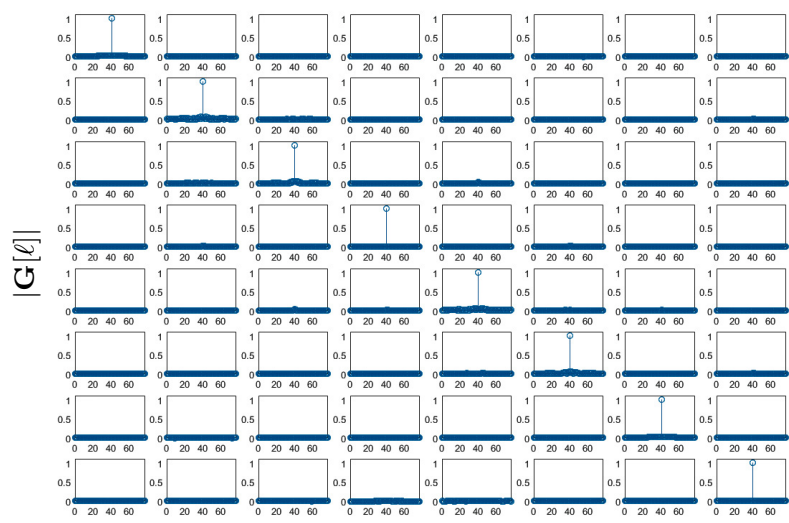

time index $\ell$

Fig. 7. Example for $\mathbf{G}[\ell]$, which comprises of the OQAMtransmultiplexed channel transmission matrix $\boldsymbol{F}(z)$ and its pseudoinverse $\boldsymbol{F}^{\dagger}(z)$ for an $M=8$ channel FMBC/OQAM system including a dispersive channel $c[\nu]$ of length 4 .

Example. For an $M=8$ channel FBMC/OQAM system with a dispersive channel $c[\nu]$ of length 4 , the system $\boldsymbol{F}(z)$ is of rank $\frac{M}{2}=4$. For the resulting pseudoinverse, the $\frac{M}{2}$ finite singular values do not possess zeros close to the unit circle, such that the impact of $\epsilon_{2}$ is insignificant. The resulting multiplication of $\boldsymbol{F}(z)$ with its pseudo-inverse $\boldsymbol{W}(z)=\boldsymbol{F}^{\dagger}(z)$ is shown in Fig. 6; the result is not an identity matrix due to the rank deficiency of $\boldsymbol{F}(z)$. However, when including the OQAM pre- and post-processing, and considering the MIMO channel matrix between the input $\mathbf{d}[\ell]$ and $\hat{\mathbf{d}}[\ell]$, such that $\hat{\mathbf{d}}[\ell]=\mathbf{G}[\ell] * \mathbf{d}[\ell]$, the system in Fig. (7) emerges, which is now free of ISI and ICI.

\section{CONCLUSIONS}

We have analysed an FBMC/OQAM system, which is known to suffer from ISI and ICI between at least adjacent subchannels in presence of a dispersive channel impulse response. We have proposed a polynomial matrix formulation for an equivalent channel transfer function, which includes the filter bank components and channel, and attempted the equalisation of this system by means of polynomial matrix algebra. Since the matrix is rank deficient by default, we have introduced a polynomial pseudo-inverse to address this problem. The equalisation leads to solution, that together with OQAM pre- and postprocessing can eliminate ISI and ICI.

\section{REFERENCES}

[1] P. Siohan, C. Siclet, and N. Lacaille. Analysis and design of OFDM/OQAM systems based on filterbank theory. IEEE Transactions on Signal Processing, 50(5):1170-1183, May 2002.

[2] M. Schellmann, Z. Zhao, H. Lin, P. Siohan, N. Rajatheva, V. Luecken, and A. Ishaque. FBMC-based air interface for 5G mobile: Challenges and proposed solutions. In 9th IEEE International Conference on Cognitive Radio Oriented Wireless Networks and Communications, pp. 102-107, Oulu, Finland, June 2014.

[3] C. Kim, Y. H. Yun, K. Kim, and J.-Y. Seol. Introduction to QAM-FBMC: From waveform optimization to system design. IEEE Communications Magazine, 54(11):66-73, Nov. 2016.

[4] B. Farhang-Boroujeny. OFDM versus filter bank multicarrier. IEEE Signal Processing Magazine, 28(3):92-112, Mar. 2011.

[5] A. I. Pérez-Neira, M. Caus, Z. Rostom, D. Le Ruyet, E. Kofidis, M. Haardt, X. Mestre, and Y. Cheng. MIMO signal processing in offset-QAM based filter bank multicarrier systems. IEEE Transactions on Signal Processing, 64(21):5733-5762, Nov. 2016.

[6] J. Fang, Z. You, I.-T. Lu, J. Li, and R. Yang. Comparisons of filter bank multicarrier systems. In IEEE Long Island Systems, Applications and Technology Conference, Farmingdale, NY, May 2013.

[7] T. Ihalainen, T. H. Stitz, M. Rinne, and M. Renfors. Channel equalization in filter bank based multicarrier modulation for wireless communications. EURASIP Journal on Advances in Signal Processing, 2007(1):049389, 2006.

[8] D. S. Waldhauser and J. A. Nossek. MMSE equalization for bandwidth-efficient multicarrier systems. In IEEE International Symposium on Circuits and Systems, pages 4-pp. Kos, Greece, May 2006.

[9] D. S. Waldhauser, L. G. Baltar, and J. A. Nossek. MMSE subcarrier equalization for filter bank based multicarrier systems. In IEEE 9th Workshop on Signal Processing Advances in Wireless Communications, pp. 525-529, Recife, Brazil, July 2008.

[10] D. S. Waldhauser, L. G. Baltar, and J. A. Nossek. Adaptive equalization for filter bank based multicarrier systems. In IEEE International Symposium on Circuits and Systems, pp. 30983101. Seattle, WA, May 2008.

[11] A. Ikhlef and J. Louveaux. Per subchannel equalization for mimo FBMC/OQAM systems. In IEEE Pacific Rim Conference on Communications, Computers and Signal Processing, pages 559-564. Victoria, Canada, Aug. 2009.

[12] L. G. Baltar, D. S. Waldhauser, and J. A. Nossek. MMSE subchannel decision feedback equalization for filter bank based multicarrier systems. In IEEE International Symposium on Circuits and Systems, pp. 2802-2805, Taipei, Taiwan, May 2009.

[13] M. Caus and A. Perez-Neira. Multi-stream transmission for highly frequency selective channels in MIMO-FBMC/OQAM 
systems. IEEE Transactions on Signal Processing, 62(4):786796, February 2014.

[14] X. Mestre and D. Gregoratti. A parallel processing approach to filterbank multicarrier MIMO transmission under strong frequency selectivity. In IEEE International Conference on Acoustics, Speech and Signal Processing, pages 8078-8082, May 2014.

[15] A. Gilloire and M. Vetterli. Adaptive filtering in subbands with critical sampling: analysis, experiments, and application to acoustic echo cancellation. IEEE Transactions on Signal Processing, 40(8):1862-1875, Aug. 1992.

[16] B. B. A. Viholainen and M. Huchard. PHYDYAS physical layer for dynamic access and cognitive radio, prototype filter and structure optimization.

[17] S. Mirabbasi and K. Martin. Overlapped complex-modulated transmultiplexer filters with simplified design and superior stopbands. IEEE Transactions on Circuits and Systems II: Analog and Digital Signal Processing, 50(8):456-469, Aug. 2003.

[18] S. Redif, J. McWhirter, and S. Weiss. Design of FIR paraunitary filter banks for subband coding using a polynomial eigenvalue decomposition. IEEE Transactions on Signal Processing, 59(11):5253-5264, Nov. 2011.

[19] J. G. McWhirter. An algorithm for polynomial matrix SVD based on generalised Kogbetliantz transformations. In 18th European Signal Processing Conference, pp. 457-461, Aalborg, Denmark, Aug. 2010.

[20] J. Foster, J. McWhirter, M. Davies, and J. Chambers. An algorithm for calculating the QR and singular value decompositions of polynomial matrices. IEEE Transactions on Signal Processing, 58(3):1263-1274, March 2010.

[21] J. G. McWhirter, P. D. Baxter, T. Cooper, S. Redif, and J. Foster. An EVD Algorithm for Para-Hermitian Polynomial Matrices. IEEE Transactions on Signal Processing, 55(5):2158-2169, May 2007.

[22] S. Redif, S. Weiss, and J. McWhirter. Sequential matrix diagonalization algorithms for polynomial EVD of parahermitian matrices. IEEE Transactions on Signal Processing, 63(1):8189, Jan. 2015.

[23] S. Weiss, A. Millar, and R. W. Stewart. Inversion of parahermitian matrices. In 18th European Signal Processing Conference, pp. 447-451, Aalborg, Denmark, Aug. 2010.

[24] B. Widrow and E. Walach. Adaptive Inverse Control. Prentice Hall, Englewood Cliffs, New York, 1995.

[25] G. H. Golub and C. F. Van Loan. Matrix Computations. John Hopkins University Press, Baltimore, Maryland, 3rd edition, 1996.

[26] S. Haykin. Adaptive Filter Theory. Prentice Hall, Englewood Cliffs, 2nd edition, 1991. 